\title{
New insights into the links between anti-diabetes drugs and gut microbiota
}

\author{
Ruixin Hu, Yanting Yuan, Chaolong Liu, Ji Zhou, Lixia Ji and Guohui Jiang \\ School of pharmacy, Qing Dao University, Qingdao, China
}

Correspondence should be addressed to L Ji or G Jiang : lixiaji@163.com or 13370830026@163.com

\begin{abstract}
In patients with type 2 diabetes mellitus (T2DM), the intestinal flora is out of balance and accompanied by leaky gut. The flora is characterized by an increase in mucus-degrading bacteria and a decrease in fiber-degrading bacteria. Short-chain fatty acids (SCFAs), as the major fiber-degrading bacteria fermentation, not only ameliorate the leaky gut, but also activate GPR43 to increase the mass of functional pancreatic $\beta$-cells and exert antiinflammation effect. At present, the gut microbiota is considered as the potential target for anti-diabetes drugs, and how to reverse the imbalance of gut microbiota has become a therapeutic strategy for T2DM. This review briefly summarizes the drugs or compounds that have direct or potential therapeutic effects on T2DM by modulating the gut microbiota, including biguanides, isoquinoline alkaloids, stilbene and C7Naminocyclic alcohols.
\end{abstract}

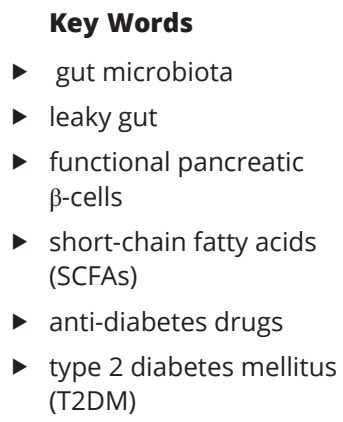
(SCFAs)

- anti-diabetes drugs

- type 2 diabetes mellitus (T2DM)

\section{Introduction}

A genome-wide association study in Chinese population has shown that there is marked reduction in microbiota diversity in patients with T2DM. The main flora of the T2DM patients is characterized by an increase in mucusdegrading bacteria and a decrease in the number of fiberdegrading bacteria. Compared with healthy people, the abundance of fiber-degrading bacteria Bifidobacterium, Faecalibacterium prausnitzii, Roseburia and Bacteroides vulgatus decreased significantly, while the mucusdegrading bacteria Akkermansia muciniphila, Bacteroides caccae, Clostridium and Escherichia coli increased in patients with T2DM $(1,2,3)$.

In the intestinal epithelial cells, the mature mucus layer is characterized by the fact that the number of fiberdegrading bacteria is significantly higher than that of mucus-degrading bacteria, so that the mucus layer has a complete barrier function (4). For example, when mice are given a diet rich in dietary fiber, fiber-degrading bacteria emerge as the times require. The fiber-degrading bacteria can produce a large number of enzymes to depolymerize and ferment dietary polysaccharides, turning them into SCFAs that can be absorbed by the host. The energy supply of the intestinal flora comes from dietary fiber or glycoproteins in the mucus layer covering the intestinal epithelium, and energy is conserved. In a diet lacking dietary fiber, the flora needs to erode the glycoproteins of mucus layer to obtain energy, leading to the number of mucus-degrading bacteria much higher than that of fiberdegrading bacteria in the intestinal tract. At the same time, a low-fiber diet leads to a sharp increase in the number of gram-negative bacteria in the intestine, which will increase the content of lipopolysaccharide (LPS) and pathological organisms. LPS and pathological organisms will take the opportunity to adhere to the thinning mucosal layer, and pass through the intestinal epithelial layer through the following mechanisms: (i) LPS and pathological organisms activate TLR2/4 receptor and recruit MyD-88 aptamers. (ii) LPS and pathological organisms combine with Nod1. (iii) LPS and pathological organisms are phagocytosed and co-localized by dendritic cells.

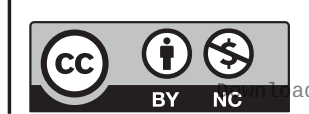

This work is licensed under a Creative Commons Attribution-NonCommercial 4.0 International License. ded from Bioscientifica.com at 04/26/2023 01:49:32PM 
(iv) LPS and pathological organisms destroy the tight junctions of intestinal epithelial cells so that tight junction proteins (occludin and zonula occludens-1) and CB2 are reduced (5). Through the previous methods, they penetrate the intestinal mucosal barrier with enhanced permeability, smoothly transfer to the lamina propria and submucosa, and continue to transfer to the circulatory system and peripheral tissues. This translocation of LPS and pathological organisms in the intestine is called 'leaky gut' (6). Bacterial DNA was found in the blood, omental, subcutaneous and mesenteric adipose tissue in T2DM patients. A small amount of bacterial DNA can increase the expression of pro-inflammatory factors in subcutaneous adipocytes. As a 'barrier' for bacteria to enter the body from the intestine, mesenteric adipose tissue has the highest bacterial diversity and number compared to other adipose tissues. The most convincing evidence is that living bacteria are detected by catalyzed reporter deposition fluorescence in situ hybridization in the subcutaneous adipose tissue and the bacteria are encapsulated in the adipose tissue (7). In addition, the tight junctions are damaged, and the mRNA levels of occludin and zonula occludens-1 (zo-1) are significantly downregulated in diabetic mice. Clinical studies have confirmed that the fecal zonulin concentration of patients with T2DM is significantly higher than that of the normal population. High-fat-diet (HFD) mice and T2DM patients have elevated serum LPS levels. The mRNA levels of the inflammatory markers PAI-1, IL- 1, TNF- $\alpha$ in mesenteric adipose tissue and the mature macrophage marker F4/80 in the subcutaneous adipose depot of mice increased (8). The previous evidence strongly indicates that the occurrence of T2DM is accompanied by the ongoing leaky gut.

In obesity or the early stage of T2DM, systemic insulin resistance can trigger a compensatory response, increase the workload of pancreas and enhance the insulin secretion by pancreas $\beta$-cells. Some evidence have proved that pancreas $\beta$-cells can be stimulated into a compensatory state by high-fat diet (HFD) with the upregulation of free fatty acid receptor 2 gene (Ffar2) in mice (9). Free fatty acid receptor 2 (FFAR2), also known as GPR43, is a subclass of the nutrition-sensitive receptor GPCR, and SCFAs are considered to be the endogenous ligands (10). In addition to the acetate existing in the blood, there is a small part of the binding form of SCFAs in the intestine. Most of them exist in an ionized state and require special transporters across the epithelial barrier. Leaky gut provides a new way for SCFAs to cross the barrier. Animal studies have shown that (i) In islets, FFAR2 can be activated by SCFAs, increasing the glucose-stimulated insulin secretion in mice through the Goq/11 signaling pathway $(11,12)$, (ii) In endocrine L cells of colon, FFAR2 mediates the secretion of GLP-1 by coupling with the ligand SCFAs, which in turn promotes the proliferation of functional islet $\beta$-cells mass $(13,14)$. Besides, SCFAs can play a role in anti-inflammation and maintaining the integrity of the intestinal barrier.

The intestinal flora of patients with T2DM is seriously out of balance, and the mucus-degrading bacteria and LPS producing pathogenic bacteria are dominant. LPS and pathological organisms transfer through the intestinal epithelial cells, transfer to the submucosa through leaky gut to the submucosa, then into the mesenteric adipose tissue, and finally into the blood and peripheral tissues. The increase of fiber-degrading bacteria is accompanied by the increase of SCFAs, which strengthen the intestinal barrier function, reduces the degree of metabolic endotoxemia and improve the function of islet $\beta$-cells. This review summarizes the research progress of Biguanides, Isoquinoline alkaloids, Stilbene, C7N-aminocyclic alcohols on intensifying intestinal barrier and improving islet $\beta$-cells function by regulating microbiota dysbiosis.

\section{Drugs}

\section{Biguanides}

Recent studies have shown that metformin can modulate the abundance of gut microbiota (Table 1). After metformin treatment on obese mice, the total amount of SCFAs in feces increased; the number of Akkermansia muciniphila, Clostridium cocleatum and the phylum Bacteroidetes elevated; the number of Prevotella, an opportunistic pathogen, reduced $(15,16)$. In T2DM patients treated with metformin, the abundance of Escherichia, Roseburia, Faecalibacterium, Bifidobacterium increased (17). Roseburia, Faecalibacterium, Bifidobacterium belonged to fiber-degrading bacteria. The number of Clostridium, Intestinibacter species and six OTUS abundances of Bacteroides reduced $(18,19)$.

As to the flora regulated by metformin, the number of fiber-degrading bacteria increased in patients, the mucusdegrading microbiota Clostridium cocleatum abundance increased in mice and decreased in patients. Prevotella had the ability to degrade mucin oligosaccharides, and its abundance reduced in HFD mice. The animal experiment treated with metformin showed that the mRNA expression for the canonical factors of the TLR/NF- $\mathrm{KB}$ signaling pathway (including TLR2, TLR4 and TNF- $\alpha$ ) 
Table 1 Drugs microbiota and the mechanism of ameliorating leaky gut (the chemical structure of the drug is drawn by ChemDraw).

\begin{tabular}{ll}
\hline Drugs & $\begin{array}{c}\text { Changes in gut microbiota } \\
\text { Escherichia, } \uparrow \text { Roseburia, } \\
\uparrow \text { Faecalibacterium, } \\
\uparrow \text { Bifidobacterium, } \\
\downarrow \text { Clostridium, } \downarrow \text { Intestinibacter } \\
\downarrow \text { Bacteroides } \\
\uparrow \text { Clostridium cocleatum, } \\
\uparrow \text { Akkermansia muciniphila } \\
\uparrow \text { Bacteroidetes } \\
\downarrow \text { Prevotella }\end{array}$ \\
\end{tabular}<smiles>COc1ccc2cc3c(cc2c1OC)CCc1cc2c(cc1-3)OCO2</smiles>

Resveratrol<smiles>Oc1ccc(/C=C/c2cc(O)cc(O)c2)cc1</smiles>

Acarbose

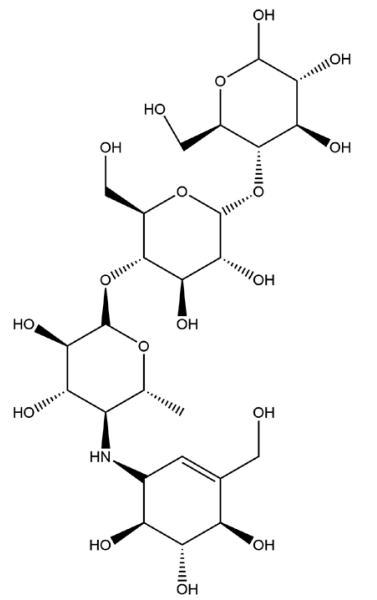
Shigella, $\uparrow$ Enterobacter, $\uparrow$ Akkermansia muciniphila, $\uparrow$ Escherichia coli, $\downarrow$ Clostridiaceae, $\downarrow$ Prevotella, $\downarrow$ Streptococcus

$\uparrow$ Bacteroides, $\uparrow$ Blautia, $\uparrow$ Parabacteroides, $\uparrow$ Ruminococcus, $\uparrow$ Bifidobacterium, $\downarrow$ Enterococcus faecalis, $\downarrow$ Proteobacteria, $\downarrow$ Akkermansia muciniphila

$\uparrow$ Faecalibacterium, $\uparrow$ Prevotella, $\uparrow$ Bifidobacterium longum, $\uparrow$ Lactobacillus gasseri, $\downarrow$ Enterococcus faecalis, $\downarrow$ Clostridium

$\uparrow$ Bacteroides, $\uparrow$ Blautia, $\uparrow$ Bifidobacterium, $\downarrow$ Desulfovibrio, $\downarrow$ Ruminiclostridium $\uparrow$ Bacteroidetes, $\uparrow$ Escherichia-

\begin{tabular}{|c|c|}
\hline Model & Mechanism \\
\hline Human & $\begin{array}{l}\text { The mRNA expression for the } \\
\text { canonical factors of the TLR/ } \\
\text { NF- } \mathrm{B} \text { s signaling pathway } \\
\text { (including TLR2, TLR4 and } \\
\text { TNF- } \alpha \text { ) downregulated. }\end{array}$ \\
\hline Mice & $\begin{array}{l}\text { The mRNA and protein } \\
\text { expression } \\
\text { of occludin and zo-1 were } \\
\text { upregulated. }\end{array}$ \\
\hline
\end{tabular}

Mice $\quad$ The level of serum LPS reduced. The protein expression of TLP2/4 and TNF- $\alpha$ was downregulated.

Mice The mRNA expression of zo-1, zo-2, occludin, claudin-1, JAM-A, the major mucin gene (MUC1, MUC2, MUC3) and transcription factor (Relm $\beta$, Reg3 $\gamma$ ) elevated. The expression of inflammatory factors IL-6, IL-16, IL-1 $\beta$ and TNF- $\alpha$, MCP- 1 decreased.

Human

$(22,23,24,25,26)$

$(27,28,29)$

Mice downregulated. The mRNA expression and protein level of tight junction-related factors occludin and zo-1 were upregulated, Transmission electron microscopy indicated the reduced gap between intestinal epithelial cells $(15,16)$. Metformin can adjust the flora in the right direction, along with restoring the normal permeability of intestinal epithelium and protecting the intestinal barrier structure. In addition, Metformin microbiota is also related to the improvement of islet $\beta$-cells function. The reduction in abundance of multiple OTUs in Bacteroides could increase the homeostasis model assessment- $\beta$ value (HOMA- $\beta$ ), indicating that the islet $\beta$-cells function was simultaneously enhanced (19). Bifidobacterium could reduce the serum levels of pro-inflammatory factors such 
as IL- $1 \beta$ and TNF- $\alpha$, and effectively prevent the occurrence of insulin resistance. After metformin treatment, the decrease in Clostridium abundance and the increase of Bifidobacterium abundance in adolescence were negatively correlated with $\mathrm{HbA}_{1 \mathrm{c}}$ levels $(20,21)$.

\section{Isoquinoline alkaloids}

In obese mice, the total content of SCFAs in feces increased after berberine treatment. The concentration of butyrate increased from $6 \mu \mathrm{g} / \mathrm{mL}$ to $12 \mu \mathrm{g} / \mathrm{mL}$. The composition of the phylum Bacteroidetes increased and the number of fiber-degrading bacteria Escherichia, Shigella, Enterobacter, Akkermansia muciniphila elevated $(22,23)$. Berberine could reduce the abundance of Clostridiaceae, Prevotella, and Streptococcus, and increase gram-negative bacteria like Escherichia coli. Prevotella and Escherichia coli were both mucus-degrading microbiota $(24,25)$.

In intestinal epithelial cells, berberine could play a similar role to metformin. Berberine targeted intestinal microbiota could downregulate TLP2/4 and TNF- $\alpha$ protein levels in T2DM mice. The tight junction zo-1 protein staining strongly marked the cytoplasm of intestinal epithelial cells and made the brush border markers uniform distributed in diabetic mice. Berberine also reduced the serum LPS level and increase the height of the intestinal mucosa in diabetic mice (26). On the whole, the characteristic of berberine on gut flora is that the abundance of fiber-degrading bacteria is higher than that of mucus-degrading bacteria. In addition, berberine microbiota has the potential to enhance the function of islet $\beta$-cells. Treatment with berberine alone on mice could decrease TG level, while HDL showed the opposite tread (23). In clinic, the decrease in TG/HDL ratio not only predicted dyslipidemia, but also indicated the occurrence of insulin resistance to some extent. Increasing the TG/HDL ratio implied that the drug had the effect of inhibiting insulin resistance. Studies revealed that Clostridiaceae, Prevotella and Streptococcus were positively correlated with the homeostasis model assessment of insulin resistance (HOMA-IR) index (25). Lowing HOMA-IR index could alleviate insulin resistance in patients with T2DM.

\section{Stilbene}

Fecal microbiota of mice receiving resveratrol (RSV) flora transplantation was characterized by increased abundance of fiber-degrading bacteria Bacteroides, Blautia, Parabacteroides, Ruminococcus and Bifidobacterium. The abundance of Enterococcus faecalis, Proteobacteria and mucus-degrading bacteria Akkermansia muciniphila decreased $(27,28)$. The existence of a large number of Proteobacteria indicated that the gut flora is in an imbalanced state (29).

Compared with HFD mice, mice treated with RSV flora not only had more complete intestinal morphology and lower intestinal permeability under a fluoroscopy electron microscope, but also showed upregulated mRNA levels of the key markers of the intestine integrity zo-1, zo- 2 , occludin, claudin- 1 and JAM-A. Relm $\beta$ secreted by goblet cells was beneficial for enhancing intestinal barrier function and integrity. The RSV flora could significantly elevate the mRNA expressions of major mucin genes (MUC1, MUC2, MUC3) and transcription factors $(\operatorname{Re} \operatorname{lm} \beta, \operatorname{Reg} 3 \gamma)$ in mice, which were close to normal levels. RSV flora could also reduce the serum levels of inflammatory factors IL-6, IL-16, IL-1 $\beta$, TNF- $\alpha$ and lowgrade inflammation marker MCP-1 to close to normal levels (27). The previous results suggest that RSV flora can reinforce intestinal structural integrity and mucosal barrier function, reduce the occurrence of inflammation and affect islet $\beta$-cells function. Experiments illustrated that transplanting resveratrol-treated mice fecal flora into HFD mice could improve the blood glucose homeostasis and insulin resistance (30). Indeed, the fasting blood glucose decreased, hyperinsulinemia improved, and the area under the curve in oral glucose tolerance test and insulin tolerance test decreased significantly. The previously described illustrated that resveratrol-induced fecal microbiota could reduce blood glucose level and improve insulin sensitivity. In addition, the HOMA-IR index value of HFD mice was twice that of mice received fecal transplantation from resveratrol-treated mice (27).

\section{C7N-aminocyclic alcohols}

In obese mice, acarbose treatment could increase the content of butyrate and the abundance of fiber-degrading bacteria Bacteroides, Blautia and Bifidobacterium, while the number of Desulfovibrio, Ruminiclostridium decreased (31). In T2DM patients, the abundance of Faecalibacterium, Prevotella, Bifidobacterium longum, Lactobacillus gasseri increased. The number of Enterococcus faecalis and mucusdegrading bacteria Clostridium abundance reduced $(32,33)$.

Acarbose also has the potential to regulate the imbalance of the flora. Due to lack of relevant research, the relationship between acarbose-regulated flora and leaky gut is unknown. However, evidence revealed that the acarbose had the effect of enhancing pancreatic islet $\beta$-cell function. The level of glycosylated hemoglobin decreased in patients treated with acarbose, which was 
associated with increased abundance of Bifidobacterium longum and Lactobacillus gasseri. Decreasing the abundance of Enterococcus faecalis resulted in a decrease in LPS level, thus preventing the progression of chronic low-grade inflammation and systemic insulin resistance $(34,35)$.

\section{Discussion}

It is well known that a long-term high-fat/low-fiber diet can easily induce the occurrence of T2DM. Due to the lack of fiber intake, patient's flora is out of balance and characterized by the predominance of pathogenic and mucus-degrading bacteria. Over time, a large number of mucus-degrading bacteria obtain energy to survive by degrading the mucus layer of the intestinal epithelium. LPS, produced by gram-negative pathogenic bacteria in intestine, can transfer through the intestinal epithelium into intestinal cells and even in peripheral tissues (adipose, skeletal muscle, liver), causing severe metabolic inflammation. Living bacteria was also detected in blood and omental, subcutaneous and mesenteric adipose tissue in diabetic patients. Both LPS and living bacteria can directly promote the M1-like polarizaiton of macrophages in visceral adipose tissue and even islets, causing systemic insulin resistance and functional decline of $\beta$-cells.

Whether it is diet therapy or drug intervention, intestinal flora is an important site to alleviate the progression of diabetes, which is closely related to metabolic inflammation, insulin resistance and islet function. Experiments showed that changes in dietary conditions could cause rapid fluctuations in the abundance of the flora. For example, the abundance of Akkermansia muciniphila, Bacteroides ovatus, Bacteroides caccae and Eubacterium rectale changed significantly when the mice equipped with human sequencing flora were subjected to daily fiber-free/fiber-rich alternating diet (4). However, the adjustments to the diet require personalized customization, which will not be discussed in this review. The experiment observed that the mucus layer of the intestinal epithelium of gram-free mice was thinner than that of normal mice, indicating that microbial colonization is a necessary factor for maintaining the thickness of the mucus layer. The serum levels of LPS and TNF- $\alpha$ decreased in obese mice receiving antibiotic treatment, suggesting that flora regulation can improve the metabolic inflammatory state. In summary, as the mucus layer of intestine is damaged, some targeted drugs would be the first choice to modulate the imbalanced flora.
This review mainly focuses on the drugs against T2DM by regulating intestinal microecology and flora. For metformin, its therapeutic effects and adverse reactions are involved in microorganisms. The analysis of intestinal genome samples of patients with T2DM after taking metformin showed a significant increase in the abundance of Escherichia. With the increase of the abundance of Escherichia coli, the increase in virulence factor LPS will lead to metabolic inflammation and an increase of white blood cell count. In addition, the increased hydrogen production by Escherichia coli promoted the production of propionate and butyrate to maintain blood sugar steady state, but it might also facilitate the synthesis of hydrogen sulfide by sulfate-reducing bacteria, causing adverse reactions such as abdominal distension and intestinal discomfort (18). After metformin treatment, increased Akkermansia used glycosylated proteins in the epithelial mucus layer as the main carbon and nitrogen sources to produce SCFAs, especially acetate and propionate, during the fermentation process. Some researchers believed that Akkermansia was beneficial to maintain the functional integrity of the intestinal mucosa, that could be used to explain the effect of metformin on improving glucose tolerance. The amount of Akkermansia in T2DM is controversial. Previous experiments suggested that the abundance of Akkermansia decreased in patients with T2DM. Akkermansia could strengthen the integrity of the intestinal epithelium by adhering to the intestinal epithelium without causing inflammation in the body. In order to study the direct effect of Akkermansia, HFD mice were given living Akkermansia orally and showed marked increase in the thickness of the mucus layer and a decrease in the incidence of endotoxemia. In contrast, a metagenomic study illustrated that Akkermansia abundance in patients with T2DM elevated. As far as I am concerned, I agree with the latter conclusion. First of all, most of the intestinal flora in the human are identified through genomics rather than cultured in vitro. Therefore, whether Akkermansia, existing in the complex human environment, can play the previously mentioned role remains to be further studied. Secondly, it is assumed that Akkermansia in vivo is beneficial to maintain the integrity of intestinal epithelium and improve intestinal permeability. The mechanism of muciniphila-derived EVs induced AMPK phosphorylation in a dose-dependent manner, resulting in upregulation of occludin expression in the intestinal epithelium, and ameliorating the loss of barrier integrity induced by LPS (36). However, there are more than one mechanism for cunning pathological microorganisms to increase intestinal permeability, and the increase in Akkermansia abundance

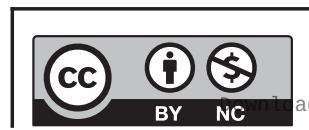

This work is licensed under a Creative Commons Attribution-NonCommercial 4.0 International License. ded from Bioscientifica.com at 04/26/2023 01:49:32PM 
cannot completely prevent leaky gut. Finally, the flora of T2DM patients is characterized by an increased abundance of most gram-negative pathogenic bacteria. The number of Akkermansia belonging to the genus gram-negative bacteria is far less than that of pathogenic bacteria. The latest research indicated that the presence of living bacteria detected in subcutaneous adipose tissue of T2DM patients further confirmed the previous conclusion. In addition to metformin, berberine can also increase the abundance of Akkermansia. Sutterella, Allisonella, Akkermansia, Bacteroides and Paraprevotella that existed in healthy male volunteers before the drug intervention were participants in the gastrointestinal side effects after the intervention, leading to an increase in the incidence of gastrointestinal adverse reactions (37). Akkermansia and Bacteroides in patients are known in T2DM patients, and the drugs mentioned in the review all have different degrees of gastrointestinal adverse reactions.

In summary, drug-targeted flora could correct the imbalance of the gut flora in T2DM patients and alleviate the leaky gut, so as to improve insulin resistance and indirectly enhance the function of islet $\beta$-cells. At present, with the exception of metformin, the relationship between the flora regulated by other drugs and adverse reactions is not clear. Moreover, the powerful evidence used to prove leaky gut was the presence of living bacteria in adipose tissue. Whether the drug-regulated flora can reduce the number of live bacteria in adipose tissue needs to be proved by further research. The code of the relationship between the drug and the flora requires a lot of experiments to unravel, which can then minimize the incidence of adverse reactions as the drug exerting a therapeutic effect.

\section{Declaration of interest}

The authors declare that there is no conflict of interest that could be perceived as prejudicing the impartiality of this review.

\section{Funding}

This work was supported by grants from the Introduced Talent Project of Qingdao University (80402010104/063-06301104).

\section{References}

1 Qin J, Li Y, Cai Z, Li S, Zhu J, Zhang F, Liang S, Zhang W, Guan Y, Shen $\mathrm{D}$, et al. A metagenome-wide association study of gut microbiota in type 2 diabetes. Nature 2012490 55-60. (https://doi. org/10.1038/nature11450)

2 Larsen N, Vogensen FK, van den Berg FW, Nielsen DS, Andreasen AS, Pedersen BK, Al-Soud WA, Sorensen SJ, Hansen LH \& Jakobsen M. Gut microbiota in human adults with type 2 diabetes differs from non-diabetic adults. PLoS ONE 20105 e9085. (https://doi. org/10.1371/journal.pone.0009085)
3 Leiva-Gea I, Sanchez-Alcoholado L, Martin-Tejedor B, CastellanoCastillo D, Moreno-Indias I, Urda-Cardona A, Tinahones FJ, Fernandez-Garcia JC \& Queipo-Ortuno MI. Gut microbiota differs in composition and functionality between children with type 1 diabetes and MODY2 and healthy control subjects: a case-control study. Diabetes Care 201841 2385-2395. (https://doi.org/10.2337/dc18-0253)

4 Desai MS, Seekatz AM, Koropatkin NM, Kamada N, Hickey CA, Wolter M, Pudlo NA, Kitamoto S, Terrapon N, Muller A, et al. A dietary fiber-deprived gut microbiota degrades the colonic mucus barrier and enhances pathogen susceptibility. Cell 20161671339 e21-1353.e21. (https://doi.org/10.1016/j.cell.2016.10.043)

5 Hasain Z, Mokhtar NM, Kamaruddin NA, Mohamed Ismail NA, Razalli NH, Gnanou JV \& Raja Ali RA. Gut microbiota and gestational diabetes mellitus: a review of host-gut microbiota interactions and their therapeutic potential. Frontiers in Cellular and Infection Microbiology 202010 188. (https://doi.org/10.3389/fcimb.2020.00188)

6 Camilleri M. Leaky gut: mechanisms, measurement and clinical implications in humans. Gut 201968 1516-1526. (https://doi. org/10.1136/gutjnl-2019-318427)

7 Cani PD, Bibiloni R, Knauf C, Waget A, Neyrinck AM, Delzenne NM $\&$ Burcelin R. Changes in gut microbiota control metabolic endotoxemia-induced inflammation in high-fat diet-induced obesity and diabetes in mice. Diabetes 200857 1470-1481. (https://doi. org/10.2337/db07-1403)

8 Massier L, Chakaroun R, Tabei S, Crane A, Didt KD, Fallmann J, von Bergen M, Haange SB, Heyne H, Stumvoll M, et al. Adipose tissue derived bacteria are associated with inflammation in obesity and type 2 diabetes. Gut 202069 1796-1806. (https://doi.org/10.1136/ gutjnl-2019-320118)

9 Priyadarshini M, Navarro G \& Layden BT. Gut microbiota: FFAR reaching effects on islets. Endocrinology 2018159 2495-2505. (https://doi.org/10.1210/en.2018-00296)

10 Priyadarshini M, Wicksteed B, Schiltz GE, Gilchrist A \& Layden BT. SCFA receptors in pancreatic beta cells: novel diabetes targets? Trends in Endocrinology and Metabolism 201627 653-664. (https://doi. org/10.1016/j.tem.2016.03.011)

11 Villa SR, Priyadarshini M, Fuller MH, Bhardwaj T, Brodsky MR, Angueira AR, Mosser RE, Carboneau BA, Tersey SA, Mancebo H, et al. Loss of free fatty acid receptor 2 leads to impaired islet mass and beta cell survival. Scientific Reports 20166 28159. (https://doi.org/10.1038/ srep28159)

12 Priyadarshini M, Villa SR, Fuller M, Wicksteed B, Mackay CR, Alquier T, Poitout V, Mancebo H, Mirmira RG, Gilchrist A, et al. An acetate-specific GPCR, FFAR2, regulates insulin secretion. Molecular Endocrinology 201529 1055-1066. (https://doi.org/10.1210/me.20151007)

13 Psichas A, Sleeth ML, Murphy KG, Brooks L, Bewick GA, Hanyaloglu AC, Ghatei MA, Bloom SR \& Frost G. The short chain fatty acid propionate stimulates GLP-1 and PYY secretion via free fatty acid receptor 2 in rodents. International Journal of Obesity 2015 39 424-429. (https://doi.org/10.1038/ijo.2014.153)

14 Miao XY, Gu ZY, Liu P, Hu Y, Li L, Gong YP, Shu H, Liu Y \& Li CL. The human glucagon-like peptide-1 analogue liraglutide regulates pancreatic beta-cell proliferation and apoptosis via an AMPK/mTOR/ P70S6K signaling pathway. Peptides 201339 71-79. (https://doi. org/10.1016/j.peptides.2012.10.006)

15 Lee $\mathrm{H} \&$ Ko G. Effect of metformin on metabolic improvement and gut microbiota. Applied and Environmental Microbiology 201480 5935-5943. (https://doi.org/10.1128/AEM.01357-14)

16 Zhang W, Xu JH, Yu T \& Chen QK. Effects of berberine and metformin on intestinal inflammation and gut microbiome composition in db/db mice. Biomedicine and Pharmacotherapy 2019 118 109131. (https://doi.org/10.1016/j.biopha.2019.109131)

17 de la Cuesta-Zuluaga J, Mueller NT, Corrales-Agudelo V, VelásquezMejía EP, Carmona JA, Abad JM \& Escobar JS. Metformin is associated with higher relative abundance of mucin-degrading Akkermansia

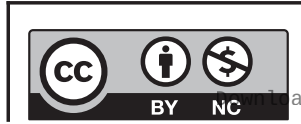

This work is licensed under a Creative Commons Attribution-NonCommercial 4.0 International License. ded from Bioscientifica.com at 04/26/2023 01:49:32PM 
muciniphila and several short-chain fatty acid-producing microbiota in the gut. Diabetes Care 201740 54-62. (https://doi.org/10.2337/ dc16-1324)

18 Forslund K, Hildebrand F, Nielsen T, Falony G, Le Chatelier E, Sunagawa S, Prifti E, Vieira-Silva S, Gudmundsdottir V, Pedersen HK, et al. Disentangling type 2 diabetes and metformin treatment signatures in the human gut microbiota. Nature 2015528 262-266. (https://doi.org/10.1038/nature15766)

19 Tong X, Xu J, Lian F, Yu X, Zhao Y, Xu L, Zhang M, Zhao X, Shen J, $\mathrm{Wu}$ S, et al. Structural alteration of gut microbiota during the amelioration of human Type 2 diabetes with hyperlipidemia by metformin and a traditional Chinese herbal formula: a multicenter, randomized, open label clinical trial. mBio 20189 3. (https://doi. org/10.1128/mBio.02392-17)

20 Karlsson FH, Tremaroli V, Nookaew I, Bergstrom G, Behre CJ, Fagerberg B, Nielsen J \& Backhed F. Gut metagenome in European women with normal, impaired and diabetic glucose control. Nature 2013498 99-103. (https://doi.org/10.1038/nature12198)

$21 \mathrm{Wu}$ H, Esteve E, Tremaroli V, Khan MT, Caesar R, Manneras-Holm L, Stahlman M, Olsson LM, Serino M, Planas-Felix M, et al. Metformin alters the gut microbiome of individuals with treatment-naive type 2 diabetes, contributing to the therapeutic effects of the drug. Nature Medicine 201723 850-858. (https://doi.org/10.1038/nm.4345)

22 Habtemariam S. Berberine pharmacology and the gut microbiota: a hidden therapeutic link. Pharmacological Research 2020155104722. (https://doi.org/10.1016/j.phrs.2020.104722)

23 Wang Y, Shou JW, Li XY, Zhao ZX, Fu J, He CY, Feng R, Ma C, Wen BY, Guo F, et al. Berberine-induced bioactive metabolites of the gut microbiota improve energy metabolism. Metabolism: Clinical and Experimental $2017 \mathbf{7 0}$ 72-84. (https://doi.org/10.1016/j. metabol.2017.02.003)

24 Yue SJ, Liu J, Wang AT, Meng XT, Yang ZR, Peng C, Guan HS, Wang CY \& Yan D. Berberine alleviates insulin resistance by reducing peripheral branched-chain amino acids. American Journal of Physiology: Endocrinology and Metabolism 2019316 E73-E85. (https:// doi.org/10.1152/ajpendo.00256.2018)

25 Shan CY, Yang JH, Kong Y, Wang XY, Zheng MY, Xu YG, Wang Y, Ren HZ, Chang BC \& Chen LM. Alteration of the intestinal barrier and GLP2 secretion in berberine-treated type 2 diabetic rats. Journal of Endocrinology 2013218 255-262. (https://doi.org/10.1530/JOE-130184)

26 Sun H, Wang N, Cang Z, Zhu C, Zhao L, Nie X, Cheng J, Xia F, Zhai H \& Lu Y. Modulation of microbiota-gut-brain axis by berberine resulting in improved metabolic status in high-fat diet-fed rats. Obesity Facts 20169 365-378. (https://doi.org/10.1159/000449507)

27 Wang P, Li D, Ke W, Liang D, Hu X \& Chen F. Resveratrol-induced gut microbiota reduces obesity in high-fat diet-fed mice. International Journal of Obesity 202044 213-225. (https://doi.org/10.1038/s41366019-0332-1)
28 Sung MM, Kim TT, Denou E, Soltys CLM, Hamza SM, Byrne NJ, Masson G, Park H, Wishart DS, Madsen KL, et al. Improved glucose homeostasis in obese mice treated with resveratrol is associated with alterations in the gut microbiome. Diabetes 201766 418-425.

29 Shin NR, Whon TW \& Bae JW. Proteobacteria: microbial signature of dysbiosis in gut microbiota. Trends in Biotechnology 201533 496-503. (https://doi.org/10.1016/j.tibtech.2015.06.011)

30 Kim TT, Parajuli N, Sung MM, Bairwa SC, Levasseur J, Soltys CM, Wishart DS, Madsen K, Schertzer JD \& Dyck JRB. Fecal transplant from resveratrol-fed donors improves glycaemia and cardiovascular features of the metabolic syndrome in mice. American Journal of Physiology: Endocrinology and Metabolism 2018315 E511-E519. (https://doi.org/10.1152/ajpendo.00471.2017)

31 Xu GD, Cai L, Ni YS, Tian SY, Lu YQ, Wang LN, Chen LL, Ma WY \& Deng SP. Comparisons of effects on intestinal short-chain fatty acid concentration after exposure of two glycosidase inhibitors in mice. Biological and Pharmaceutical Bulletin 201841 1024-1033. (https:// doi.org/10.1248/bpb.b17-00978)

32 Su B, Liu H, Li J, Sunli Y, Liu B, Liu D, Zhang P \& Meng X. Acarbose treatment affects the serum levels of inflammatory cytokines and the gut content of bifidobacteria in Chinese patients with type 2 diabetes mellitus. Journal of Diabetes 20157 729-739. (https://doi. org/10.1111/1753-0407.12232)

33 Zhang X, Fang Z, Zhang C, Xia H, Jie Z, Han X, Chen Y \& Ji L. Effects of acarbose on the gut microbiota of prediabetic patients: a randomized, double-blind, controlled crossover trial. Diabetes Therapy 20178 293-307. (https://doi.org/10.1007/s13300-0170226-y)

34 Gu Y, Wang X, Li J, Zhang Y, Zhong H, Liu R, Zhang D, Feng Q, Xie X, Hong J, et al. Analyses of gut microbiota and plasma bile acids enable stratification of patients for antidiabetic treatment. Nature Communications 20178 1785. (https://doi.org/10.1038/s41467-01701682-2)

35 Smith BJ, Miller RA, Ericsson AC, Harrison DC, Strong R \& Schmidt TM. Changes in the gut microbiome and fermentation products concurrent with enhanced longevity in acarbose-treated mice. BMC Microbiology 201919 130. (https://doi.org/10.1186/ s12866-019-1494-7)

36 Chelakkot C, Choi Y, Kim DK, Park HT, Ghim J, Kwon Y, Jeon J, Kim MS, Jee YK, Gho YS, et al. Akkermansia muciniphila-derived extracellular vesicles influence gut permeability through the regulation of tight junctions. Experimental and Molecular Medicine 201850 e450. (https://doi.org/10.1038/emm.2017.282)

37 Bryrup T, Thomsen CW, Kern T, Allin KH, Brandslund I, Jorgensen NR, Vestergaard H, Hansen T, Hansen TH, Pedersen O, et al. Metformin-induced changes of the gut microbiota in healthy young men: results of a non-blinded, one-armed intervention study Diabetologia 201962 1024-1035. (https://doi.org/10.1007/s00125019-4848-7)

Received in final form 9 December 2020

Accepted 17 December 2020

Accepted Manuscript published online 18 December 2020 https://ec.bioscientifica.com https://doi.org/10.1530/EC-20-0431 (c) 2021 The authors Published by Bioscientifica Ltd

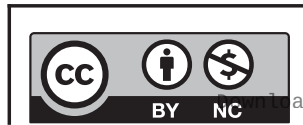

This work is licensed under a Creative Commons Attribution-NonCommercial 4.0 International License. ded from Bioscientifica.com at 04/26/2023 01:49:32PM 